\title{
Birefringence, Photo luminous, Optical Limiting and Third Order Nonlinear Optical Properties of Glycinium Phosphite (GIP) Single Crystal: A potential Semi Organic Crystal for Laser and Photonics Applications
}

\author{
Rajesh Krishnan ${ }^{a}$, Mani Ayyanar $^{b}$, Anandan Kasinathan ${ }^{a}$, Praveen Kumar $^{b}$ \\ ${ }^{a}$ Department of Physics, AMET University, Kanathur, 603112, India \\ ${ }^{b}$ Department of Physics, Presidency College, Chennai, 600005, India
}

Received: March 30, 2017; Revised: October 14, 2017; Accepted: November 23, 2017

\begin{abstract}
Glycinium Phosphite $(G l P)$ has been synthesized and characterized successfully. The fluorescence spectrum of the compound showed one broad peak at $282 \mathrm{~nm}$. Linear absorption value was calculated by Optical Limiting method. Birefringence study was carried out and the birefringence of the crystal is found to be depending on the wavelength in the entire visible region. Third order nonlinear optical property of the crystal was carried out by Z-Scan technique and non linear refractive index and third order susceptibility was calculated. Thermal stability of the crystal was found using TG and DTA thermal analyzer and the results shows that the crystals have good thermal stability. The crystal was also analyzed by photo conductivity analyzer to determine the optical conductivity of the crystal.
\end{abstract}

Keywords: Optical Limiting, Thermal, Z-Scan, Photo luminous, birefringence.

\section{Introduction}

Extensive research have been carried out on organic Nonlinear optical materials for laser applications ${ }^{1-3}$, frequency doubling services ${ }^{4}$, opto-electronics and fiber optic communications ${ }^{5}$, optical information and optical data storage devices $^{6}$. Due to the presence of $\pi-\pi$ bonds in the organic materials provides an excellent nonlinear optical property, which intern satisfies the needs of the above mentioned technological applications. A large number of materials have been found to exhibit laser induced refractive index changes such as fullerenes, liquid crystals, and organic materials and their derivatives. Among organic materials, Amino acids and their derivatives have recently attracted much attention due to their high tendency to crystallize in asymmetric structure and for their synthetic flexibility that can offer the modification of nonlinear properties. The nonlinear optical properties of this type of materials can be improved by following suitable synthetic procedures to design molecules with delocalize $\pi$ electrons, donor-accepter-donor and acceptor-donor-acceptor properties $^{7-9}$. Semi organic materials plays and major role in many potential NLO applications instead of organic materials. The NLO properties and optical limiting behaviors of semi organic materials are enhanced by combining the organics with the inorganic. From the various classes of organic compounds, Glycine based material plays a major role in the optoelectronics and photonics applications. Many Glycine derivative organic materials exhibit interesting NLO efficiency ${ }^{10,11}$. Under the perturbation of the external electric field this conjugation of $\pi$-electron was affected by electron donor and acceptor group, which results the higher rate of NLO property. Glycine is the simplest amino acid; it forms many useful new compounds with other organic as well as inorganic materials. Glycine Phosphite is one of the interesting semi organic materials in glycine derivative series. The structure of Glycine Phosphite was reported by Averbuch-Pouchot ${ }^{12}$. In our previous publication on the GIP crystal we reported the electrical and mechanical properties of the material ${ }^{13}$. are In the present research, we are focusing on various optical properties of the crystal such as photoconductivity, birefringence, photo luminous, optical limiting and third order NLO efficiency of the crystal for the first time. Thermal behaviors and thermal stability of crystal also discussed in this work.

\section{Experimental}

Single crystals of GlP were grown from aqueous solution by employing slow solvent evaporation technique. The source materials taken in equimolar ratio; calculated parent compounds were dissolved in double distilled water and mixed well using a magnetic stirrer continuously for 9 hours. Then the purified saturated solution was allowed to evaporate at room temperature. Synthesized salt was purified by adopting the repeated recrystallization method. The successfully recrystalized salt was further used for growing bulk crystal. Good optical quality crystal was harvested in a period of 27 days. The solubility of GlP is found to be high in aqueous solution than the other organic polar solvents; hence distilled water was selected to grow good quality crystals. The Photograph of as grown crystal of GlP is given in the figure 1 . 


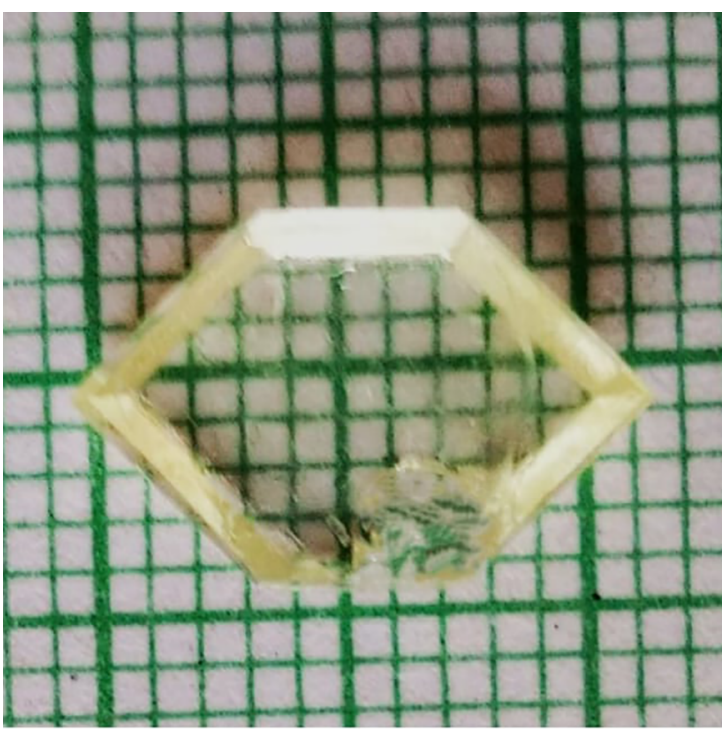

Figure 1. Photograph of GLP crystal.

\section{Result and Discussions}

\subsection{Single crystal X-ray diffraction study}

Single crystal X-ray diffraction analysis was carried out on GlP single crystal for finding the cell parameters and crystal structure of glycinium Phosphite crystal using Enraf Nonius CAD-4/MACH 3 diffractometer, with MoK $\alpha$ radiation. The glycinium Phosphite crystal is crystallize in monoclinic crystal system with the space group P2. The lattice parameters are $\mathrm{a}=9.619 \AA ; \mathrm{b}=8.467 \AA ; \mathrm{c}=7.621$ $\AA, \alpha=\gamma$ and $\beta=100.11$. Cell parameters and space group of the grown crystal found from single XRD are in good agreement with the reported value ${ }^{12}$.

\subsection{Birefringence study}

Birefringence analysis is precise technique to find the optical perfection and optical homogeneity in crystals ${ }^{14}$ phase matching angles are also can be found using birefringence ${ }^{15}$. Since, optical perfection is important characteristics of a material for which, it can be used in optoelectronics devices. The birefringence values have been calculated by finding absolute fringe orders using the relation:

$$
\Delta \mathrm{n}=\frac{\mathrm{K} \lambda}{t}
$$

Where, $\lambda$ is wave length, $t$ is the thickness of the crystal and $\mathrm{K}$ is the order of fringe. Figure 2 shows the variation of birefringence with the wavelength and it shows that the birefringence values are lies in between 0.028 to 0.084 in the wavelength ranging from $241 \mathrm{~nm}$ to $710 \mathrm{~nm}$. The slight variation in birefringence over a wide range of wavelength indicates that the crystal is suitable material for second and third harmonic generation device fabrication ${ }^{16}$. The obtained birefringence values were found to be positive integer and it is increase with increasing wavelength, which illustrated the grown $G l P$ crystal possess positive dispersion of birefringence ${ }^{17}$. A minimum dispersion in birefringence can be the key factor in frequency conversion process such as second and third harmonic generations ${ }^{18}$.

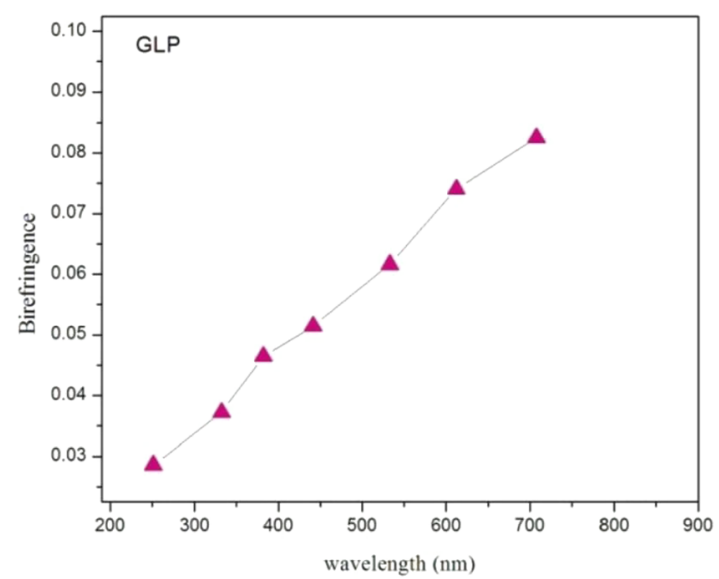

Figure 2. Plot of Birefringence verses Wavelength.

\subsection{Photo Luminous (PL) Study}

Photoluminescence (PL) is the phenomenon in which electronic states of solids are excited by light of particular energy and the excitation energy is released as light. The photon energies reflect the variety of energy states that are present in these materials. The PL spectra were recorded using a Fluoromax-4 spectrofluorometer. The fluorescence spectrum of GlP crystal is shown in figure 3. It shows that there is a strong emission peak at $282 \mathrm{~nm}$, it due to the shift of Phosphite ions from their excited state to ground state. It can be noted that the emission line at $282 \mathrm{~nm}$ shows a strong and broad bandwidth with the FWHM of $28 \mathrm{~nm}$. The maximum value of full width half maximum is an additional beneficial to the development of tunable or ultrafast lasers ${ }^{19}$

\subsection{Optical Limiting}

The increase in the usage of low power lasers in various applications needs for the design of optical limiters with high efficiency ${ }^{20}$. Linear optical absorption of the crystal was examined in the air atmosphere at room temperature $\left(\sim 28^{\circ} \mathrm{C}\right)$ by optical limiting experiment. Well cut and polished surface of the crystal is subjected to light, and the focal length is about $8 \mathrm{~cm}$.

The low intense laser light form the laser source is subjected to the sample and the output power was measured. A power meter detector is used to monitor the input and measure the output power of laser. This process is repeated for several input powers and the corresponding outputs readings were recorded. A graph is drawn between input and output power and is shown in figure 4. By finding the output power, focal length and power output of the laser source linear absorption was calculated. The linear absorption of the $G l P$ crystal was calculated to be $3.21 \mathrm{~J} / \mathrm{cm}^{-1}$.

\subsection{Thermal Analysis}

The thermal behavior of GlP crystal was measured by thermo - gravimetric (TG) and differential thermal analysis 


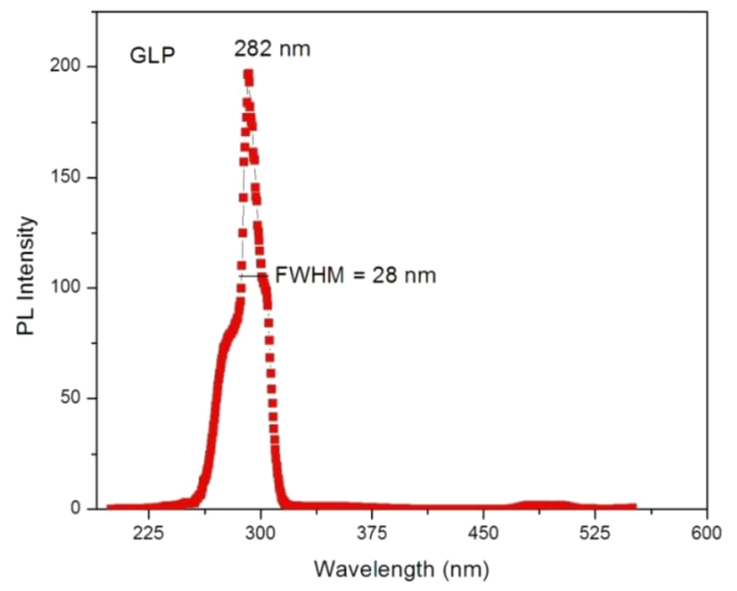

Figure 3. Emission spectrum of GLP.

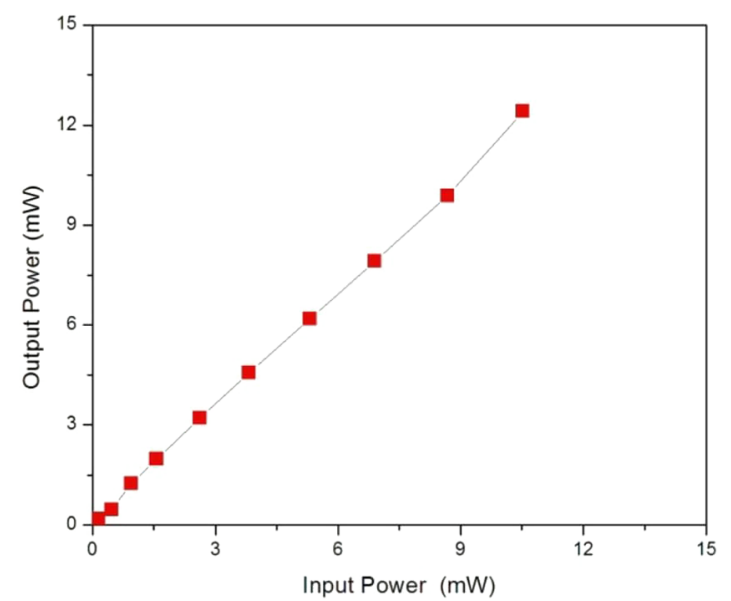

Figure 4. Optical Limiting Plot.

(DTA) by using NETZSCH STA $409 \mathrm{CCD}$ from $25^{\circ} \mathrm{C}$ to $600^{\circ} \mathrm{C}$ as heating rate of $20^{\circ} \mathrm{C} / \mathrm{min}$ in a nitrogen atmosphere. Figure 5 illustrates the changes in TG and DTA curve of GLP crystal. The DTA curve implies that the crystal undergoes an endothermic transition of two significant peaks at $154^{\circ} \mathrm{C}$ and $337^{\circ} \mathrm{C}$, where the material begins to melt. The first peak of endothermic represents the temperature at which the melting terminates which corresponds to its melting point. The TG curve of this crystal indicates that the material is stable upto to $341^{\circ} \mathrm{C}$ and above this temperature the weight loss is not due to self degradation of GlP but merely its evaporation after its melting. The sharpness of the endothermic peak shows good degree of crystallinity of the grown crystal and thermodynamically stable in all atmospheres at room temperature.

Further, by adopting the Coats-Red fern integral method $^{21}$, the obtained weight loss data at all temperatures were used to evaluate the thermal decomposition kinetics such as activation energy $\left(E_{\mathrm{a}}\right)$, enthalpy of activation $(\Delta \mathrm{H})$, entropy of activation $(\Delta \mathrm{S})$ and Gibb's free energy $\left(\Delta \mathrm{G}^{*}\right)$. The calculated values of $E a, \mathrm{~A}, \Delta \mathrm{S}, \Delta \mathrm{H}$ and $\Delta \mathrm{G}^{*}$ of the GLP crystal are presented in Table 1.

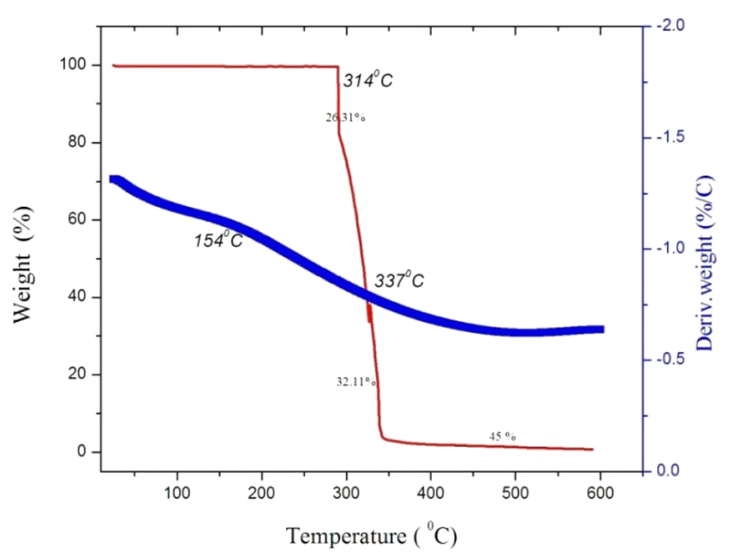

Figure 5. TG and DTA Curve of GIP.

Table 1. Kinetic parameters of $G l P$ crystal

\begin{tabular}{ccc}
\hline S.No & Kinetic parameters & Values \\
\hline 1. & Activation energy $\mathrm{E}_{\mathrm{a}}[\mathrm{kJ} / \mathrm{mol}]$ & 54.56 \\
2. & Frequency factor $\mathrm{A}\left[\mathrm{ks}^{-1}\right]$ & 5.02 \\
3. & Entropy of activation $\Delta \mathrm{S}[\mathrm{kJ} / \mathrm{mol} . \mathrm{k}]$ & -68.52 \\
4. & Enthalpy of activation $\Delta \mathrm{H}[\mathrm{kJ} / \mathrm{mol}]$ & 56.21 \\
5. & Gibb's free energy $\Delta \mathrm{G}^{*}[\mathrm{~kJ} / \mathrm{mol}]$ & 14.57 \\
\hline
\end{tabular}

From the calculated data one can noted that the enthalpy of activation is almost equal to activation energy, suggesting that $G l P$ crystal is in condensed phase between $145^{\circ} \mathrm{C}$ to $340^{\circ} \mathrm{C}$. The positive value of $\Delta \mathrm{G}^{*}$ shows that the reaction involved in the decomposition of GlP is non spontaneous and the decomposition occurred in a single step after the melting point. The thermal and kinetic data present in this study may help to find out new applications based on GlP crystal in the field of quality control, purity analysis,,$^{22,23}$ and thermal decomposition optimization.

\subsection{Third order nonlinear optical measurement}

The nonlinear refractive index of the materials was determined by the Z-scan technique ${ }^{24}$. This technique based on the fact that the intensity of light changes along the axis of the convex lens and is maximum at the focus. Hence, by moving the sample through the focus, the nonlinear refraction can be estimated by observing the spot size variation at the plane of finite aperture and detector combination. The experiment was done at an air-cooled Ar ion laser beam of $488 \mathrm{~nm}$ and $514 \mathrm{~nm}$ wavelength with an average power of $10 \mathrm{~mW}$. The laser beam was focused to a beam waist of $20 \mu \mathrm{m}$ with a lens of $5 \mathrm{~cm}$ focal length. The transmission for the samples was measured with aperture and without aperture in the far-field of the lens, as the sample moved through the focal point.

Figure 6 gives the normalizing transmission curve for the open aperture. The transmission is symmetric with respect to the exact focus $(Z=0)$, where transmission is minimum. This indicate that the sample exhibits reverse saturation 
absorption, $\mathrm{RSA}^{25}$. The calculated nonlinear refractive index and absorption coefficient of $G l P$ crystal are $4.21 \times 10^{-7} \mathrm{~cm}^{2} / \mathrm{W}$ and $5.62 \times 10^{4} \mathrm{~cm} / \mathrm{W}$, respectively. Third order non linear optical Susceptibility $\left(\chi^{3}\right)$ was calculated and it take the value of $2.325 \times 10^{-4}$ esu.

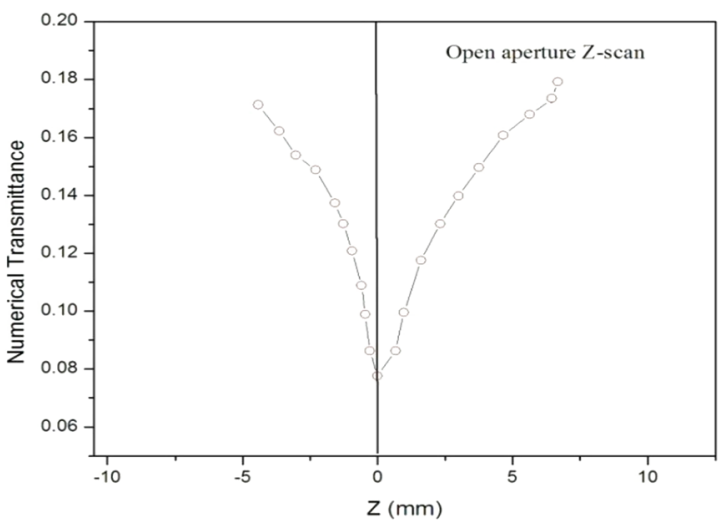

Figure 6. Z-Scan Open aperture.

\subsection{Photo conductivity study}

Photoconductivity property of the GlP crystal was carried out using Keithley 485 picoammeter. The experiment was performed at room temperature. Electrical contacts between parallel plates were made at a spacing of about $0.11 \mathrm{~cm}$ on the samples using silver coatings. The Direct Current (DC) input was increased gradually and the corresponding dark currents were measured from the electrometer. The sample was illuminated with a halogen lamp of $100 \mathrm{~W}$ powers and the corresponding photocurrent was recorded for the applied voltage. The variations of photocurrent $\left(\mathrm{I}_{\mathrm{p}}\right)$ and dark current $\left(\mathrm{I}_{\mathrm{d}}\right)$ with applied voltage are shown in Figure 7 . Both photo current and dark current of GLP crystal increase linearly with applied field. It is observed from the plot that the dark current is less than photocurrent, thus suggesting that GlP exhibits positive photoconductivity. In general positive photoconductivity is attributed to generation of mobile charge carriers caused by the absorption of photons ${ }^{26}$.

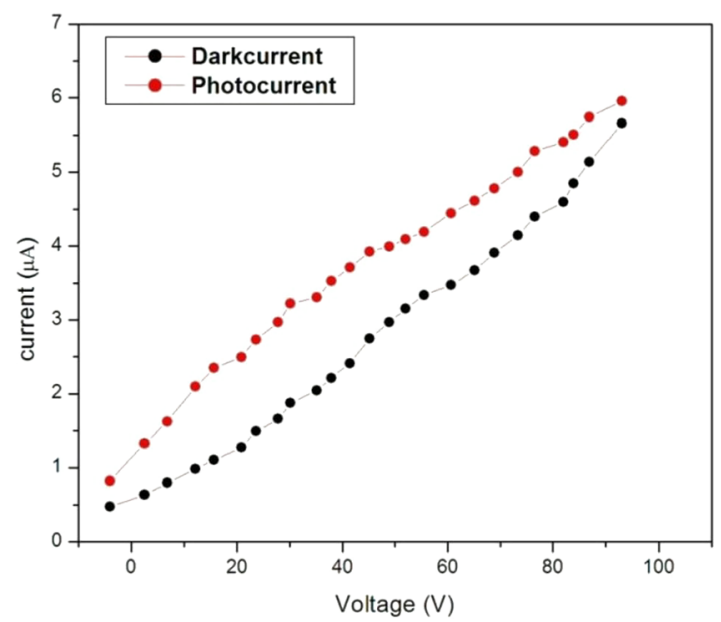

Figure 7. Photoconductivity curve.

\section{Conclusions}

Semi organic Glycinium Phosphite (GlP) single crystal was grown by slow evaporation technique with good optical quality. The crystal crystallizes in monoclinic crystal system. Solubility of the crystal is high in aqueous solution than polar solvents. The minimum dispersion of the birefringence in the visible region suggests that the crystal is suitable for second and third order harmonic generation frequency conversions. Emission spectra shows that there is sharp peak at $282 \mathrm{~nm}$ with broad full width half maximum value. This suggests the crystal can be useful in ultrafast lasers. Optical limiter has been used to find out the linear absorption coefficient of the crystal. The linear absorption of the GlP crystal was found to be $3.21 \mathrm{~J} / \mathrm{cm}^{-1}$. The crystal is thermally stable upto $341{ }^{\circ} \mathrm{C}$, this shows good degree of crystallinity of the grown crystal and it is thermodynamically stable in all atmospheres at room temperature. Activation energy, enthalpy of activation and Gibb's Free energy of the crystal was calculated using the thermal data. It is found that activation energy and enthalpy are almost equal it is due to the condense state of the crystal between $145^{\circ} \mathrm{C}$ to $340^{\circ} \mathrm{C}$. Gibb's free energy holds the positive value. Second and third order SHG measurement show the crystal is a well suitable material for harmonic generation efficiency operations. The calculated nonlinear refractive index and absorption coefficient of GlP crystal from the z-scan technique are $4.21 \times 10^{-7} \mathrm{~cm}^{2} / \mathrm{W}$ and $5.62 \times 10^{4} \mathrm{~cm} / \mathrm{W}$, respectively. Third order non linear optical Susceptibility $(\chi 3)$ was calculated and it take the value of $2.325 \times 10^{-4} \mathrm{esu}$. GlP crystal exhibits positive photoconductivity.

\section{References}

1. Xu D, Xue D. Chemical bond simulation of KADP single-crystal growth. Journal of Crystal Growth. 2008;310(7-9):1385-1390.

2. Ren X, Xu D, Xue D. Crystal growth of KDP, ADP and KADP. Journal of Crystal Growth. 2008;310(7-9):2005-2009.

3. Xue D, Ratajczak H. Constituent chemical bonds and nonlinear optical coefficients of $\mathrm{Na}_{2} \mathrm{SeO}_{4} \cdot \mathrm{H}_{2} \mathrm{SeO}_{3} \cdot \mathrm{H}_{2} \mathrm{O}$ molecular crystal. Chemical Physics Letters. 2003;371(5-6):601-607.

4. Anandha Babu G, Perumal Ramasamy R, Ramasamy P, Krishna Kumar V. Synthesis, Crystal Growth, and Characterization of an Organic Nonlinear Optical Donor- $\pi$-Acceptor Single Crystal: 2-Amino-5-nitropyridinium-Toluenesulfonate. Crystal Growth \& Design. 2009;9(7):3333-3337.

5. Xue D, Zhang S. Chemical bond analysis of the correlation between crystal structure and nonlinear optical properties of complex crystals. Physica B: Condensed Matter. 1999;262(1-2):78-83.

6. Mohan Kumar R, Rajan Babu D, Jayaraman D, Jayavel R, Kitamura K. Studies on the growth aspects of semi-organic L-alanine acetate: a promising NLO crystal. Journal of Crystal Growth. 2005;275(1-2):e1935-e1939. 
7. Vijayakumar S, Babu M, Kalluraya B, Chandrasekharan K. Third-order nonlinear optical response of newly synthesized accepter/donor substituted propylidene aryloxy acet hydrazide. Optik - International Journal for Light and Electron Optics. 2012;123(1):21-25.

8. Serbutoviez C, Bosshard C, Knopfle G, Wyss P, Pretre P, Guenter P, et al. Hydrazone Derivatives, an Efficient Class of Crystalline Materials for Nonlinear Optics. Chemistry of Materials. 1995;7(6):1198-1206.

9. Albota M, Beljonne D, Brédas JL, Ehrlich JE, Fu JY, Heikal AA, et al. Design of Organic Molecules with Large Two-Photon Absorption Cross Sections. Science. 1998;281(5383):1653-1656.

10. Lenin M, Chandrasekar M, Udhayakumar G. Growth and characterization of nonlinear optical single crystal: Glycine zinc sulfate. International Journal of Chem Tech Research. 2014;6(5):2683-2688.

11. Sivanesan T, Natarajan V, Pandi S. Growth and Characterization of L-Glycine Formate Single Crystal. Asian Journal of Chemistry. 2010;22(6):4229-4233.

12. Averbuch-Pouchot MT. Structures of glycinium phosphite and glycylglycinium phosphite. Acta Crystallographica Section C. 1993;C49:815-818.

13. Rajesh K, Mani A, Praveen Kumar P. Growth and Characterization of L-Glycinium Phosphate: A Promising Crystal for Opto - Electronics Applications. Mechanics, Material Science \& Engineering. 2017;9:73-78.

14. Kar S, Verma S, Bartwal KS. Structural and optical investigations on $\mathrm{Mn}$ doped $\mathrm{Li}_{2} \mathrm{~B}_{4} \mathrm{O}_{7}$ crystals. Crystal Research \& Technology. 2009;44(3):305-308.

15. Fischer DW, Ohmer MC, Schunemann PG, Pollak TM. Direct measurement of $\mathrm{ZnGeP}_{2}$ birefringence from 0.66 to $12.2 \mu \mathrm{m}$ using polarized light interference. Journal of Applied Physics. 1995;77(11):5942-5945.

16. Krishnan P, Gayathri K, Rajakumar PR, Jayaramakrishnan V, Gunasekaran S, Anbalagan G. Studies on crystal growth, vibrational, optical, thermal and dielectric properties of new organic nonlinear optical crystal: Bis (2,3-dimethoxy-10oxostrychnidinium) phthalate nonahydrate single crystal. Spectrochimica Acta Part A: Molecular and Biomolecular Spectroscopy. 2014;131:114-124.

17. Fork RL, Martinez OE, Gordon JP. Negative dispersion using pairs of prisms. Optics Letters. 1984;9(5):150-152.

18. Yariv A, Yeh P. Optical Waves in Crystals: Propagation and Control of Laser Radiation. New York: Wiley; 1984.

19. Ning K, Zhang Q, Zhang D, Fan J, Sun D, Wang X, et al. Crystal growth, characterization of $\mathrm{NdTaO}_{4}$ : A new promising stoichiometric neodymium laser material. Journal of Crystal Growth. 2014;388:83-86.

20. Dehghani Z, Nadafan M, Saievar Iranizad E. The effect of external applied fields on the third order nonlinear susceptibility of ferronematics. Journal of Molecular Liquids. 2015;204:70-75.

21. Coats AW, Redfern JP. Kinetic Parameters from Thermogravimetric Data. Nature. 1964;201(4914):68-69.

22. Giron D. Applications of Thermal Analysis and Coupled Techniques in Pharmaceutical Industry. Journal of Thermal Analysis and Calorimetry. 2002;68(2):335-357.

23. El-Ries MA, Abo-Attia FM, El-Bayoumi A, Eman GS. Thermal characterization of lefluomide. Insight Pharmaceutical Sciences. 2011;1(2):18-23.

24. Sheik-Bahae M, Said AA, Wei TH, Hagan DJ, Van Stryland EW. Sensitive measurement of optical nonlinearities using a single beam. IEEE Journal of Quantum Electronics. 1990;26(4):760-769.

25. Henari FZ, Patil PS. Nonlinear Optical Properties and Optical Limiting Measurements of \{(1Z)-(4-(Dimethylamino)Phenyl) Methylene\} 4-Nitrobenzocarboxy Hydrazone Monohydrate under CW Laser Regime. Optics and Photonics Journal. 2012;4(7):182-188.

26. Joshi VN. Photoconductivity: Art: Science and Technology. New York: Marcel Dekker; 1990. 